\title{
Interaction of Sirt3 with OGG1 contributes to repair of mitochondrial DNA and protects from apoptotic cell death under oxidative stress
}

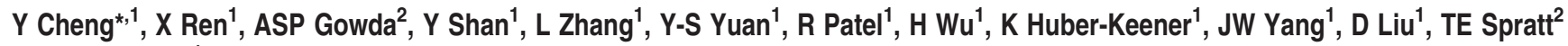 \\ and J-M Yang*,1
}

Sirtuin 3 (Sirt3), a major mitochondrial $N A D^{+}$-dependent deacetylase, targets various mitochondrial proteins for lysine deacetylation and regulates important cellular functions such as energy metabolism, aging, and stress response. In this study, we identified the human 8-oxoguanine-DNA glycosylase 1 (OGG1), a DNA repair enzyme that excises 7,8-dihydro-8-oxoguanine (8-0xOG) from damaged genome, as a new target protein for Sirt3. We found that Sirt3 physically associated with OGG1 and deacetylated this DNA glycosylase and that deacetylation by Sirt3 prevented the degradation of the OGG1 protein and controlled its incision activity. We further showed that regulation of the acetylation and turnover of OGG1 by Sirt3 played a critical role in repairing mitochondrial DNA (mtDNA) damage, protecting mitochondrial integrity, and preventing apoptotic cell death under oxidative stress. We observed that following ionizing radiation, human tumor cells with silencing of Sirt3 expression exhibited deteriorated oxidative damage of mtDNA, as measured by the accumulation of 8-oxoG and 4977 common deletion, and showed more severe mitochondrial dysfunction and underwent greater apoptosis in comparison with the cells without silencing of Sirt3 expression. The results reported here not only reveal a new function and mechanism for Sirt3 in defending the mitochondrial genome against oxidative damage and protecting from the genotoxic stress-induced apoptotic cell death but also provide evidence supporting a new mtDNA repair pathway.

Cell Death and Disease (2013) 4, e731; doi:10.1038/cddis.2013.254; published online 18 July 2013

Subject Category: Cancer Metabolism

Sirtuins are $\mathrm{NAD}^{+}$-dependent protein deacetylases analogous to the sirtuin 2 (Sirt2) enzyme in yeast. The mammalian sirtuin family consists of seven deacetylases (Sirt1-Sirt7) and is involved in the regulation of a wide range of cellular processes, including transcription, energy metabolism, insulin secretion, and stress response, through deacetylating a variety of protein targets. ${ }^{1}$ Among these sirtuins, Sirt3, a 44-kDa protein that, upon transportation into the mitochondria, is activated by cleavage of a $16-\mathrm{kDa}$ fragment, functions as a primary mitochondrial stress-responsive protein deacetylase. The importance of this mitochondrial deacetylase is evidenced by a study showing that the mice deficient in Sirt3 exhibited globally increased acetylation of the mitochondrial proteins. $^{2}$ Several substrates for Sirt3 have been identified, including aldehyde dehydrogenase $2(\mathrm{ALDH} 2),{ }^{3}$ isocitrate dehydrogenase $2\left(\right.$ IDH2) ${ }^{4}$ and glutamate dehydrogenase $(\mathrm{GDH}){ }^{5}$ and through affecting the acetylation levels of these proteins, Sirt3 can regulate mitochondrial functions such as tricarboxylic acid cycle, carbon metabolism, glycolysis, and fatty acid oxidation. For example, Sirt3-mediated deacetylation of succinate dehydrogenase subunit $A^{6}$ and IDH2, ${ }^{4}$ two enzymes involved in tricarboxylic acid cycle, results in activation of these enzymes. Sirt3 has been shown to deacetylate and activate acetyl-CoA synthetase $2^{7}$ and $\mathrm{GDH},{ }^{5}$ resulting in an upregulation of the tricarboxylic acid cycle. Also, Sirt3 can regulate the status of mitochondrial redox and oxidative phosphorylation (OXPHOS) via controlling the functions of the mitochondrial proteins..$^{8,9}$ It has been reported that deacetylation of superoxide dismutase ${ }^{10-12}$ or IDH2 $2^{5,13}$ by Sirt3 results in an increased antioxidative activity of these enzymes and a decreased level of cellular reactive oxygen species (ROS). Loss of Sirt3 causes an increase in the acetylation of complex I of the electron transport chain and a decrease of its activity, leading to a reduction of the cellular ATP level. ${ }^{14}$ In addition, Sirt3 deacetylates and inactivates cyclophilin D, causing detachment of hexokinase II from the mitochondria and stimulation of OXPHOS. ${ }^{15}$ All of these studies indicate a critical role for Sirt3 in controlling the physiological function of the mitochondria.

\footnotetext{
${ }^{1}$ Department of Pharmacology, Milton S Hershey Medical Center, Penn State Hershey Cancer Institute, Pennsylvania State University College of Medicine, 500 University Drive, Hershey, PA 17033-0850, USA and ${ }^{2}$ Department of Biochemistry and Molecular Biology, Milton S Hershey Medical Center, Penn State Hershey Cancer Institute, Pennsylvania State University College of Medicine, 500 University Drive, Hershey, PA 17033-0850, USA

${ }^{*}$ Corresponding author: J-M Yang or Y Cheng, Department of Pharmacology, Penn State College of Medicine, Penn State Hershey Cancer Institute, Pennsylvania State University College of Medicine, CH74, 500 University Drive, PO Box 850, Hershey, PA 17033-0850, USA. Tel: +717531 1630 or +7175310003×281125; Fax: + 717531 0011; E-mail: jyang2@ @mm.psu.edu or yxc24@psu.edu

Keywords: Sirt3; OGG1; mitochondria; DNA repair; apoptosis

Abbreviations: OGG1, 8-oxoguanine-DNA glycosylase 1; 8-0xoG, 7,8-dihydro-8-oxoguanine; ALDH2, aldehyde dehydrogenase 2; IDH2, isocitrate dehydrogenase 2; NAM, nicotinamide; GDH, glutamate dehydrogenase; mtDNA, mitochondrial DNA; ROS, reactive oxygen species; BER, base excision repair; CHX, cycloheximide; OXPHOS, oxidative phosphorylation

Received 06.3.13; revised 20.5.13; accepted 04.6.13; Edited by C Munoz-Pinedo
} 
The human mitochondrial DNA (mtDNA) contains the genes encoding 13 polypeptides involved in respiration and OXPHOS, two rRNAs, and a set of 22 tRNA molecules. ${ }^{16}$ Mutation of the mitochondrial genome is believed to have significant implications in various pathophysiologic processes such as aging and carcinogenesis. Moreover, the mtDNA is considerably more prone to oxidative damage than the nuclear genome. ${ }^{17}$ Oxygen-free radicals generated by the mitochondrial respiration or by exposure to ionizing radiation or chemicals predominantly cause base damage in DNA, and the most frequent mutagenic base lesion is 7 , 8-dihydro-8-oxoguanine (8-oxoG). ${ }^{18}$ This altered base can pair with $A$ as well as $C$ residues, leading to an increased frequency of spontaneous GC-AT transversion mutations. ${ }^{18,19}$ If 8 -oxoG is not repaired promptly, the accumulation of this mutagenic base in mtDNA will result in mitochondrial dysfunction and trigger apoptotic cell death.

8-Oxoguanine-DNA glycosylase 1 (OGG1) is a DNA repair enzyme executing the excision of 8-oxoG, an oxidative form of guanine and a mutagenic base generated as a result of exposure to ROS. ${ }^{20}$ The activity of OGG1 in repairing 8-oxoG within the nuclear genome is regulated by the acetylation status of this enzyme, and OGG1 is a substrate for p300, a transcriptional coactivator with histone acetyltransferase activity. ${ }^{21}$ As OGG1 is also located in the mitochondria and oxidative damage in the mtDNA is primarily repaired by the base excision repair (BER) mechanism, ${ }^{22}$ this important BER enzyme plays a pivotal role in the repair of mtDNA damage by excising 8-oxoG from the mitochondrial genome. ${ }^{23,24}$ It has been shown that the mitochondria from the OGG1-knockout mice had a significantly reduced 8-oxoG incision activity, ${ }^{25}$ and in the OGG1-deficient mice, 8-oxoG accumulated in the mtDNA at a higher rate than in wild-type mice. ${ }^{26}$

As Sirt3 is the primary mitochondrial protein deacetylase participating in the regulation of mitochondrial functions and OGG1 is a key mitochondrial BER enzyme responsible for the repair of 8 -oxoG, in this study, we sought to determine whether the acetylation status and mtDNA repair function of OGG1 are regulated by Sirt3. We found that Sirt3 and OGG1 are physically associated and OGG1 is a substrate for Sirt3. Deacetylation of OGG1 by Sirt3 contributes to the maintenance of the stability of this DNA repair enzyme and the integrity of mitochondria and limiting apoptotic death in cells subjected to oxidative stress. Our study not only reveals a new role and mechanism for Sirt3 in preserving mitochondrial function, protecting mtDNA from oxidative damage and supporting cell survival under oxidative stress, but also provides new insights into the regulatory pathways of mtDNA repair.

\section{Results}

Sirt3 deacetylates OGG1 and retains its incision activity. To determine whether Sirt3 affects acetylation status of OGG1, we used an expression plasmid to overexpress Sirt3 or an siRNA to knock down Sirt3 expression in human glioma cell line LN229. Figure 1a shows that in the cells overexpressing Sirt3, the acetylation of OGG1 was markedly reduced compared with that in the control cells transfected with an empty plasmid. By contrast, silencing of Sirt3 expression caused an increase in acetylation of OGG1. To verify the effect of Sirt3 on acetylation of OGG1, we carried out the in vitro acetylation assay using the immune-purified proteins of OGG1 and Sirt3 (Figure 1b, left panel) as the substrate and enzyme, respectively. As shown in Figure 1b, in the presence of Sirt3 and $\mathrm{NAD}^{+}$, acetylation of OGG1 was remarkably decreased compared with that in the absence of Sirt3 or $\mathrm{NAD}^{+}$. These experiments demonstrated the ability of Sirt3 to deacetylate OGG1 and provide the evidence for OGG1 as a substrate of Sirt3. To show the functional significance of the interaction between Sirt3 and OGG1, we next tested the effect of Sirt3 depletion on the incision activity of OGG1, as OGG1 is the primary DNA repair enzyme responsible for the excision of 8-oxoG. LN229 cells were transfected with a non-targeting RNA or a Sirt3-targeted RNA, and the mitochondrial extracts were prepared for the DNA cleavage assay. In this assay, 8-oxoG-containing oligonucleotides were used as substrates. As shown in Figure 1c, the amounts of cleaved fragments were significantly less in the cells transfected with a Sirt3-targeted siRNA than in the control cells transfected with a non-targeting RNA, indicating that depletion of Sirt3, which was shown to cause an increase in acetylation of OGG1 (Figure 1a), impairs the BER function of the mitochondrial OGG1.

Sirt3 physically associates with OGG1. To further explore the interaction between Sirt3 and OGG1, we performed a series of coimmunoprecipitation experiments. In these
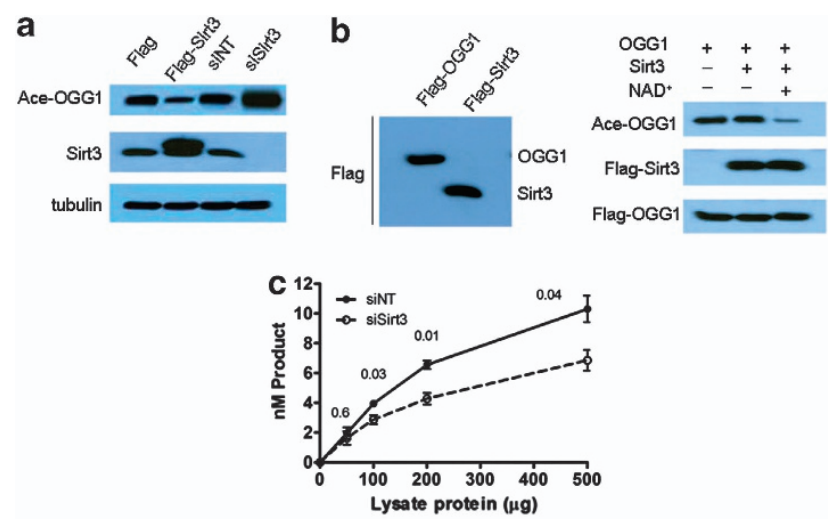

Figure 1 Effects of Sirt3 on deacetylation and incision activity of OGG1. (a) Deacetylation of OGG1 by Sirt3 in vivo: The human glioma cells LN229 were transfected with a Flag-Sirt3 expression plasmid or a Sirt3 siRNA; transfection with a Flag plasmid or a non-targeting siRNA was performed as respective controls. The levels of Sirt3 and acetyl-OGG1 were examined by western blot. Tubulin was used as a loading control. (b) Deacetylation of OGG1 by Sirt3 in vitro: Purified OGG1 and Sirt3 proteins from the HEK293T cells transfected with a Flag-OGG1 expression plasmid or a Flag-Sirt3 expression plasmid (left panel) were incubated in the presence or absence of $1 \mathrm{mM} \mathrm{NAD}^{+}$in the deacetylation assay buffer $(50 \mathrm{mM}$ Tris- $\mathrm{HCl}$ (pH 9.0), $4 \mathrm{mM} \mathrm{MgCl}_{2}, 50 \mathrm{mM} \mathrm{NaCl}, 0.5 \mathrm{mM}$ dithiothreitol) at $30^{\circ} \mathrm{C}$ for $3 \mathrm{~h}$. At the end of incubation, western blot was performed using the antibodies against acetyl-OGG1, Sirt3, or OGG1 (right panel). (c) Sirt3 knockdown inhibits the incision of 8-oxOG by OGG1: Excision of OxOG was catalyzed by mitochondria lysate. Mitochondria were isolated from LN229 cells transfected with a scrambled siRNA and Sirt3 siRNA. For OGG1 cleavage reactions, various amounts of mitochondria lysate were incubated with $20 \mathrm{nM}$ of oxoG-duplex for $30 \mathrm{~min}$ at $37^{\circ} \mathrm{C}$ and then assayed by PAGE and analyzed by Image Quant software. Each point represents mean \pm S.E. of three identical experiments, with $P$-values 
experiments, cell lysates from LN229 cell line were immunoprecipitated with an IgG or an antibody against OGG1 and then probed with an antibody to Sirt3. As shown in Figure 2a, Sirt3 was coimmunoprecipitated with an antibody against OGG1. We also immunoprecipitated Sirt3 from cell lysates and found that OGG1 was pulled down with Sirt3 but not with the IgG-negative control (Figure $2 b$ ). To validate the association of Sirt3 with OGG1, we executed similar experiments in the LN229 cells transfected with a control Flag plasmid, a Flag-tagged Sirt3 plasmid (Figure 2c) or a Flag-tagged OGG1 plasmid (Figure 2d). Figure 2c demonstrates that in the cells expressing the Flag-Sirt3, OGG1 was coimmunoprecipitated with the anti-Flag antibody. Likewise, Sirt3 was coimmunoprecipitated with the anti-Flag antibody in the cells expressing the Flag-OGG1 (Figure 2d). Together, these results displayed a previously unrecognized interaction between Sirt3 and OGG1 and identified OGG1 as a new target protein for Sirt3.

Effects of Sirt3 expression on the turnover of OGG1 protein. To understand how depletion of Sirt3 causes a reduction of the OGG1 activity, we measured and compared the expression of OGG1 protein in the cells with or without silencing of Sirt3 expression or in the cells with or without overexpression of Sirt3. Figure 3a demonstrates that OGG1 protein level was decreased when Sirt3 expression was knocked down compared with that in the control cells but was increased in the cells transfected with a Sirt3 expression

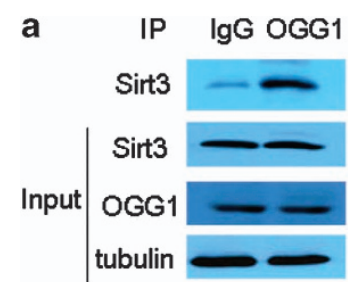

c

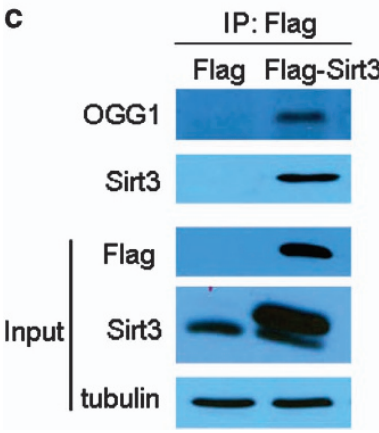

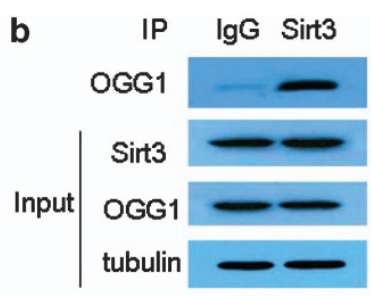

d

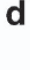

d

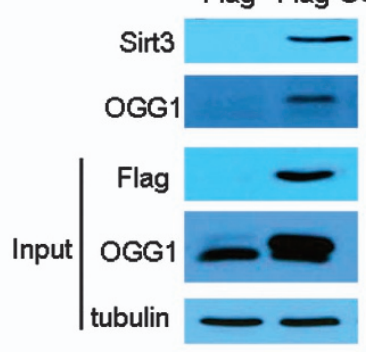

Figure 2 Physical association of Sirt3 with OGG1. (a) Extracts of LN229 cells were immunoprecipitated with a normal IgG or an OGG1 antibody and then immunoblotted with a Sirt3 antibody. (b) Extracts of LN229 cells were immunoprecipitated with a normal IgG or Sirt3 antibody and then immunoblotted with an OGG1 antibody. (c) Cell lysates of LN229 cell line transfected with a Flag-tagged Sirt3 plasmid were immunoprecipitated with a Flag antibody, and the immunoprecipitates were then subjected to western blotting with an anti-OGG1 or anti-Sirt3 antibody. (d) Cell lysates of LN229 cell line transfected with a Flag-tagged OGG1 plasmid were immunoprecipitated with a Flag antibody, and the immunoprecipitates were then subjected to western blotting with an anti-Sirt3 or anti-OGG1 antibody plasmid. A similar but more apparent effect of Sirt3 depletion on OGG1 protein amount was observed in the cellular lysates (Figure $3 b$ ) or mitochondrial extracts (Figure $3 c$ ) from the cells treated with $\gamma$-irradiation. To verify the specificity of the effect of Sirt3 on OGG1, we then determined whether the effect of knockdown of Sirt3 expression on the amount of OGG1 protein could be rescued by forced expression of this protein deacetylase. Figure $3 d$ shows that the expression of OGG1 protein was downregulated when Sirt3 expression was knocked down; however, introduction of the Sirt3 expression plasmid blocked the downregulation of OGG1 protein in the cells subjected to silencing of Sirt3 expression. These results prompted us to hypothesize that loss of Sirt3 may facilitate the turnover of the OGG1 protein, as OGG1 has been reported to be degraded by calpain, a calciumdependent cysteine protease. ${ }^{27}$ To test our hypothesis, we compared the turnover rate of OGG1 in the LN229 cells with or without silencing of Sirt3 expression by pulse chasing the OGG1 protein in the presence of cycloheximide $(\mathrm{CHX})$, an inhibitor of protein synthesis. Supporting our hypothesis, the results show that the turnover of OGG1 protein was enhanced in the cells depleted of Sirt3 expression compared with that in the control cells $\left(t_{1 / 2}=\sim 0.8\right.$ versus $\left.\sim 2 \mathrm{~h}\right)$ (Figure $3 e$ ). Next, we wanted to know whether or not the inhibitors of calpain, ALLM or E64d, could prevent the downregulation of OGG1 in the Sirt3-knockdown cells. Figure $3 f$ shows that the downregulation of OGG1 in Sirt3knockdown cells was blocked by ALLM or E64d. These results suggest that deacetylation of OGG1 by Sirt3 may hinder degradation of OGG1 by calpain, contributing to the stabilization of this DNA repair enzyme.

Silencing of Sirt3 expression aggravates the irradiationinduced mtDNA damage. To further demonstrate the importance of the Sirt3-mediated regulation of OGG1 in repairing mtDNA, we measured and compared the accumulation of the oxidized DNA marker 8-oxoG in the cells with or without depletion of Sirt3 following an irradiation treatment. Figure $4 \mathrm{a}$ demonstrates that compared with the nonirradiated cells, the irradiated cells had an accumulation of 8-oxoG, as monitored by immunostaining with an 8-oxoG antibody and observing under a fluorescence microscope. Remarkably, silencing of Sirt3 expression further increased the content of 8-oxoG in the cells exposed to irradiation. Confocal microscopy showed that 8-oxoG was mostly colocalized with MitoTracker Red, a mitochondria-selective dye (Figure 4b), indicating a mitochondrial accumulation of 8-oxoG in the irradiated cells.

The mtDNA 4977-bp deletion, also known as delta-mtDNA (4977) mutation, is the most frequent and common mtDNA mutation associated with oxidative damage ${ }^{28}$ hence, we examined and compared the effect of irradiation on occurrence of mtDNA 4977-bp deletion in the cells with or without siRNA-mediated depletion of Sirt3. Figure $4 \mathrm{c}$ shows that following irradiation, there were higher levels of delta-mtDNA (4977) mutation in the cells depleted of Sirt3 than in the control cells, as evidenced by the appearance of a 358-bp fragment of mtDNA; the amounts of the wild-type mtDNA were identical in the cells with or without silencing of Sirt3 expression (Figure $4 d$ ). These results demonstrated that 


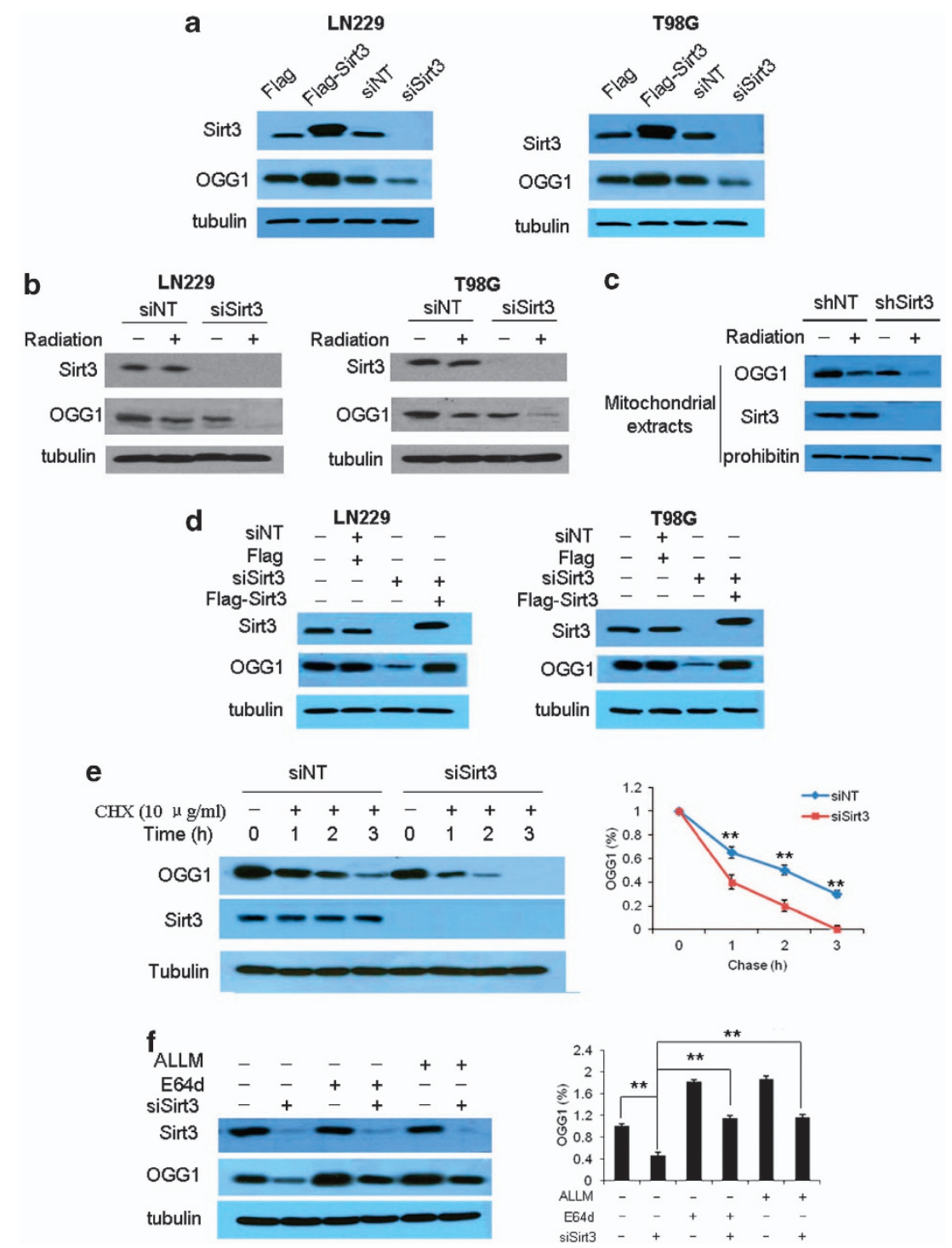

Figure 3 Silencing of Sirt3 expression promotes the degradation of OGG1 by calpain. (a) LN229 or T98G cells were transfected with a Sirt3 siRNA or a Flag-Sirt3 plasmid. The levels of OGG1 and Sirt3 were examined by western blot. Tubulin was used as a loading control. (b) LN229 or T98G cells were transfected with a non-targeting RNA or a Sirt3-targeted siRNA and then were treated or untreated with irradiation. The levels of Sirt3 and OGG1 were examined by western blot. (c) LN229 cells expressing a Sirt3 shRNA or non-targeting RNA were treated or untreated with irradiation. At the end of the treatment, the mitochondria were isolated using a mitochondrial isolation kit (Pierce). The protein levels of Sirt3 and OGG1 were examined by western blot. Prohibitin was used as a loading control. (d) LN229 or T98G cells were transfected with a Sirt3 siRNA that targets only the noncoding sequences of the Sirt3 mRNA, followed by transfection with a Flag-Sirt3 expression plasmid. The protein levels of OGG1 and Sirt3 were examined by western blot. (e) LN229 cells were transfected with a non-targeting RNA or an siRNA targeting Sirt3, followed by treatment with $10 \mu \mathrm{g} / \mathrm{ml} \mathrm{CHX}$. At the indicated time points, OGG1 protein was examined by western blot. Each point represents the mean \pm S.E. of three experiments. ${ }^{* \star} P<0.01$. (f) LN229 cells with or without silencing of Sirt3 expression were either untreated or treated with ALLM or E64d. Twenty-four hours later, the protein levels of Sirt3 and OGG1 were examined by western blot. Each bar represents the mean \pm S.E. of three experiments ${ }^{\star \star} P<0.01$

dysfunction of Sirt3 resulted in a deregulation of mtDNA repair and underscored a critical role of Sirt3 in preventing instability of the mitochondrial genome.

Silencing of Sirt3 expression deteriorates the impairment of mitochondrial integrity by irradiation. mtDNA damage, including 8-oxoG accumulation and mtDNA 4977-bp deletion, may result in mitochondrial degeneration. ${ }^{29}$ To further evaluate the consequence of the mtDNA repair deficiency caused by dysfunction of Sirt3, we assessed the effect of Sirt3 depletion on mitochondrial morphology,
OXPHOS, ROS production, and mitochondrial membrane potential $\left(\Delta \Psi_{\mathrm{m}}\right)$. Electron microscopic examination of the mitochondrial ultrastructure found that the non-targeting RNA-transfected cells untreated with $\gamma$-irradiation displayed typical shaped mitochondria with well-formed cristae; by contrast, the Sirt3-knockdown cells with or without radiation treatment and the non-targeting RNA-transfected cells treated with radiation showed morphological abnormalities of the mitochondria with distorted crista structures (Figure 5a). Comparing with the control cells, the Sirt3knockdown cells treated with irradiation had more dramatic 
a

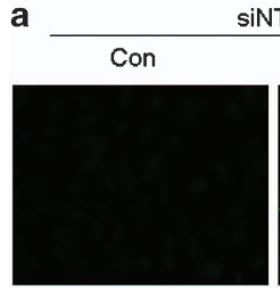

siNT

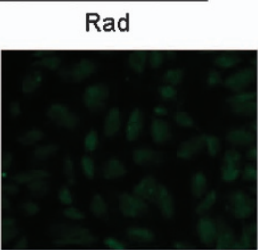

b

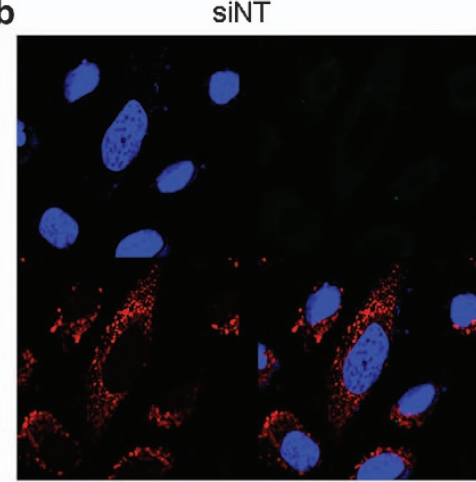

Rad

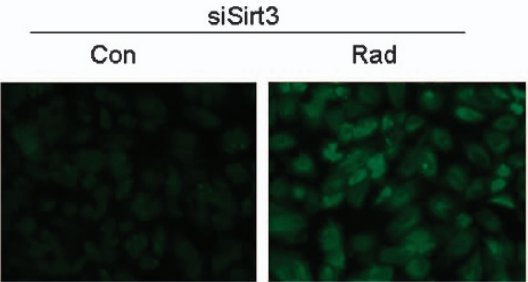

siSirt3

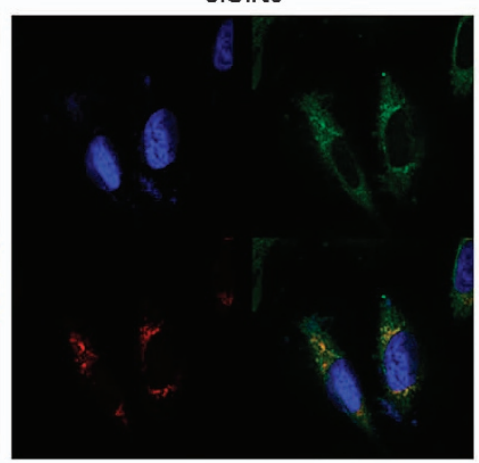

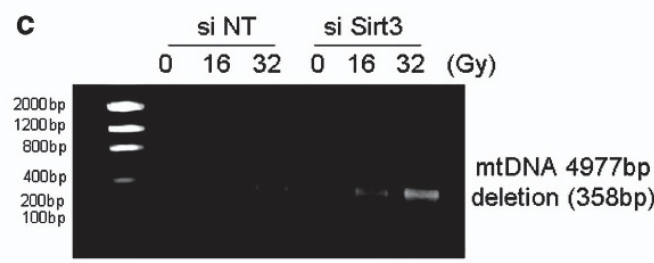

d $\quad \frac{\text { si NT }}{0} \quad$\begin{tabular}{llll}
$16 \quad 32$ & si Sirt3 \\
\hline & $16 \quad 32$
\end{tabular}

(Gy)

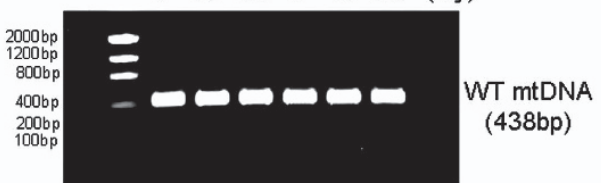

Figure 4 Silencing of Sirt3 expression increases the accumulation of 8-0xoG in the mitochondria and the mtDNA 4977 bp deletion. (a) LN229 cells with or without silencing of Sirt3 expression were treated or untreated with irradiation (16 Gy). Twenty-four hours later, the cells were immunostained with an antibody against 8-oxoG. 8-oxoG staining was visualized using a fluorescence microscope. (b) The accumulation of 8-0xoG in the mitochondria was demonstrated by its colocalization (green) with the mitochondriaselective dye, MitoTracker Red. Nuclei were counterstained with DAPI (blue). (c and d) LN229 cells were transfected with a non-targeting RNA or a Sirt3-targeted siRNA and then were treated with 16 or 32 Gy $\gamma$-irradiation. Untreated cells were used as controls. At the end of the treatment, cellular DNA was extracted, and PCR analyses of the mtDNA 4977 bp deletion (c) and the wild-type mtDNA (d) were performed

changes in the mitochondrial ultrastructure, including the appearance of swollen or hydropic mitochondria, reduction in the amount of cristae or loss of observable crista structure (Figure 5a). Oxygen consumption, which is indicative of OXPHOS, was significantly decreased in the cells subjected to silencing of Sirt3 expression compared with that in the control cells without depletion of Sirt3 (Figures $5 \mathrm{~b}$ and $\mathrm{c}$ ). In the Sirt3-knockdown cells, $\Delta \Psi_{\mathrm{m}}$ also declined significantly in comparison with that in the control cells (Figure $5 d$ ). In contrast, ROS levels were significantly higher in the Sirt3knockdown cells than in the control cells (Figure 5e). These results provide further support for an essential role of Sirt3 in protecting mitochondrial function, which might be attributed, at least partially, to the ability of this mitochondrial deacetylase to regulate the turnover of OGG1.
Activation of apoptosis by $\gamma$-irradiation can be blunted by overexpression of OGG1. Because the damage to mtDNA is a trigger of apoptosis, next we wanted to determine how Sirt3 activity affects the apoptosis induced by genotoxic effect of radiation. We first measured and compared apoptosis in the cells with or without depletion of Sirt3 following irradiation treatment. Depletion of Sirt3 augmented apoptosis in the tumor cells treated with irradiation, as evidenced by increases in the level of cleaved PARP (Figure 6a) and Annexin V staining (Figure 6b). As a downregulation of OGG1 protein was observed in the cells treated with radiation (Figures $3 b$ and $c$ ), we thus asked whether there was a causal association between the downregulation of this mtDNA repair enzyme and activation of apoptosis in the irradiated cells. We observed that forced 
a

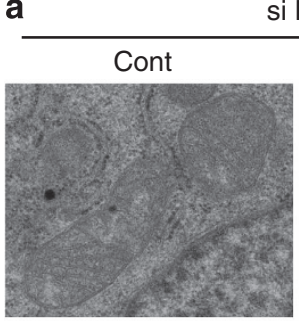

si NT

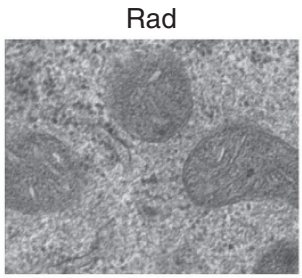

b

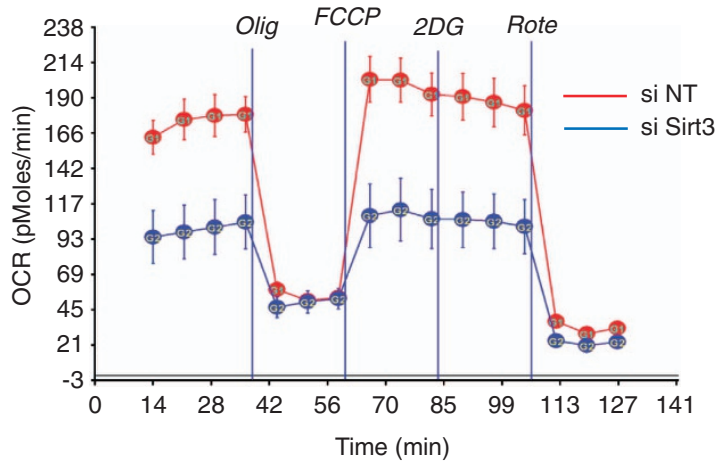

si Sirt3

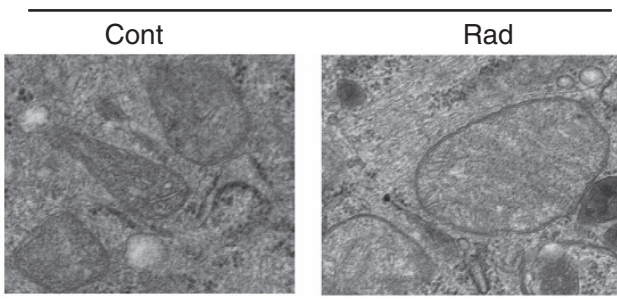

C

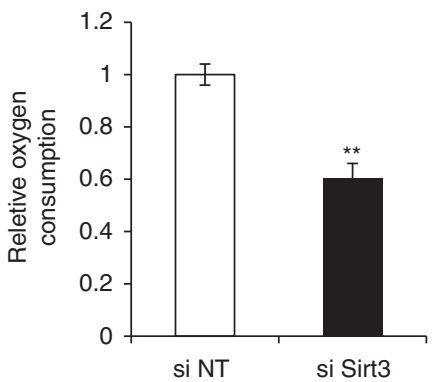

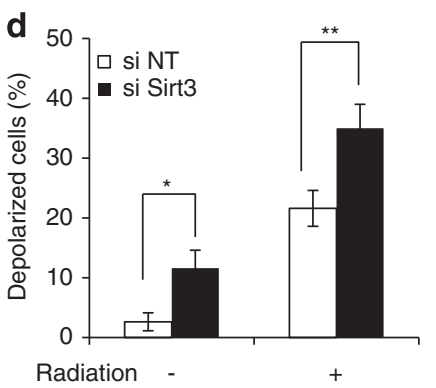

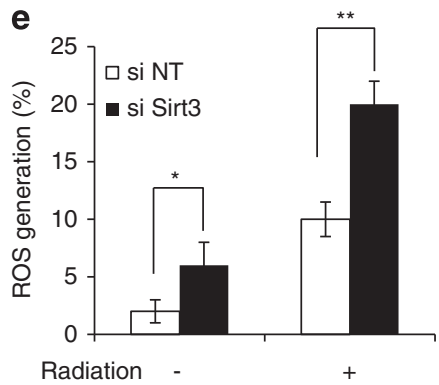

Figure 5 Knockdown of Sirt3 expression exacerbates the effects of irradiation on the mitochondrial function. (a) LN229 cells were transfected with a non-targeting RNA or a Sirt3-targeted siRNA and then were treated or untreated with irradiation (16 Gy). At the end of the treatment, the cells were harvested by trypsinization, fixed, and embedded in spur resin. Thin sections $(90 \mathrm{~nm}$ ) were cut and examined at $80 \mathrm{kV}$ with a JEOL 1200EX transmission electron microscope. (b) LN229 cells with or without silencing of Sirt3 expression in the absence of irradiation were treated sequentially as indicated with $10 \mu \mathrm{M}$ Olig, $3 \mu \mathrm{M} \mathrm{FCCP,} 1 \mathrm{M} 2 \mathrm{DG}$, and $10 \mu \mathrm{M}$ Rote. OCR was monitored using a Seahorse Bioscience Extracellular Flux Analyzer in real time (mean \pm S.D., $n=3$ ). (c) LN229 cells with or without silencing of Sirt3 expression in the absence of irradiation were collected, and oxygen consumption was measured using an oxygen electrode. Each bar represents the mean \pm S.E. of three experiments ${ }^{\star *} P<0.01$. (d) LN229 cells with or without silencing of Sirt3 expression were treated or untreated with irradiation (16 Gy), and the mitochondrial membrane potential was determined by flow cytometric analysis of JC-1 staining. Each bar represents the mean \pm S.E. of three experiments ${ }^{*} P<0.05,{ }^{* \star} P<0.01$. (e) LN229 cells with or without silencing of Sirt3 expression were treated or untreated with irradiation (16 Gy), and the level of ROS was measured by staining with DCF-DA and analyzed by flow cytometry. Each bar represents the mean \pm S.E. of three experiments ${ }^{*} P<0.05,{ }^{*} P<0.01$

expression of OGG1 markedly attenuated the apoptosis activated by radiation in the cells either with or without depletion of Sirt3 (Figure 6c), suggesting that when cells undergo stress such as ionizing radiation, downregulation of OGG1, which causes accumulation of impaired mtDNA, could be one of the mechanisms by which dysfunction of Sirt3 promotes apoptosis.

Effects of Sirt3 on growth, proliferation and clonogenicity of tumor cells. Finally, we wanted to know how Sirt3 affects tumor cell growth and survival. We compared the growth curves, proliferation rates and colony formation of LN229 cells expressing different amounts of Sirt3 (Figure 7a). These cells were cultured and grown from different clones selected after stable transfection with a Sirt3targeted shRNA. As shown in Figure 7, the tumor cells with knockdown of Sirt3 grew (Figure 7b) and proliferated
(Figure 7c) remarkably slower than the wild-type cells, and the growth and proliferations of these cells were in proportion to their expression of Sirt3. The cells with knockdown of Sirt3 expression were also much less clonogenic than the wildtype cells (Figure 7d). These results suggest that expression of Sirt3 may contribute to tumor development and progression, likely through its protective action from apoptosis.

\section{Discussion}

The roles and importance of the mtDNA repair mechanisms and pathways in the protection against carcinogenesis, aging, and other human diseases have been increasingly recognized in the past decade. This study discloses a previously unappreciated role of the mitochondrial protein deacetylase Sirt3 in alleviating oxidative damage to mtDNA and the mechanism involved. We show that dysfunction of Sirt3 
a

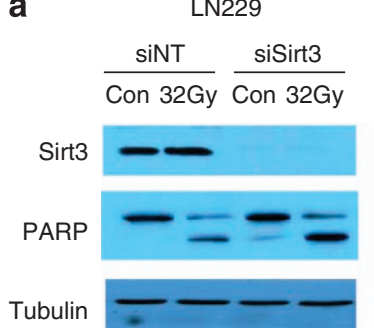

T98G

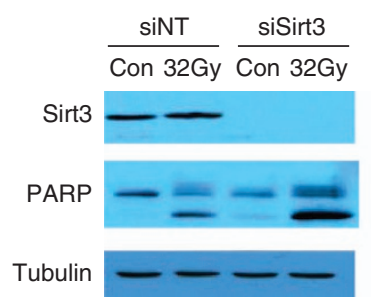

b

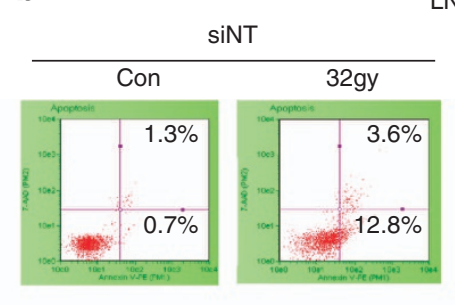

LN229

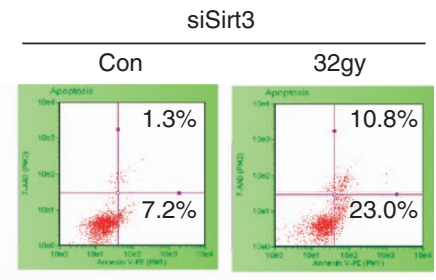

T98G
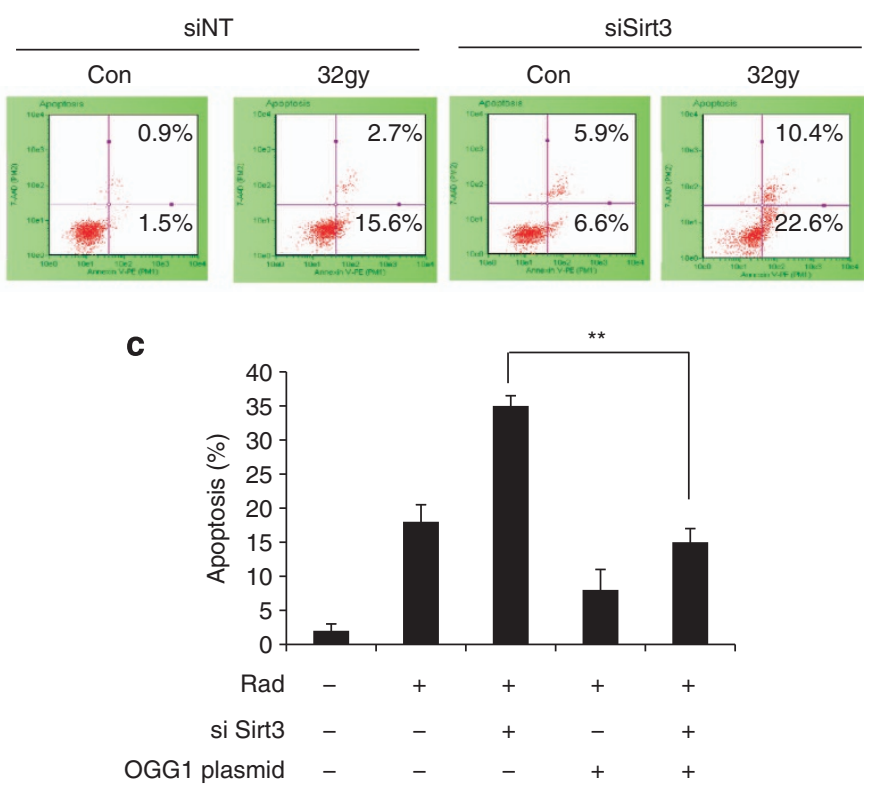
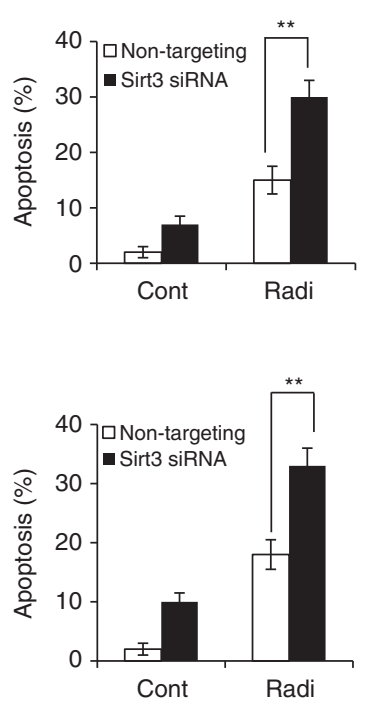

Figure 6 Enhanced apoptosis caused by Sirt3 knockdown in irradiated tumor cells can be blocked by forced expression of OGG1. (a and b) LN229 or T98G cells with or without silencing of Sirt3 expression were treated or untreated with $32 \mathrm{~Gy} \gamma$-irradiation. Apoptosis was determined by: (1) western blot of cleaved PARP. Tubulin was used as a loading control (left panels). (2) Flow cytometric analyses of Annexin staining (right panels). Each bar represents the mean \pm S.E. of three experiments ** $P<0.01$. (c) LN229 cells with or without silencing of Sirt3 expression were transfected with an OGG1 expression plasmid or a control plasmid, followed by treatment with $32 \mathrm{~Gy} \gamma$-irradiation. Apoptosis was determined by flow cytometric analyses of Annexin staining. Each bar represents the mean \pm S.E. of three experiments ${ }^{* *} P<0.01$

deteriorates the mtDNA damage and mitochondrial lesion caused by irradiation (Figures 4 and 5). We further demonstrate that Sirt3 impacts the repair of mtDNA through its ability to deacetylate OGG1, a DNA glycosylase important in BER, and that loss of Sirt3 results in increases of acetylation and degradation of OGG1 and a decrease of the incision activity of this enzyme (Figures 1 and 3 ) and promotes stress-induced apoptosis (Figure 6). Because the ROS generated by the mitochondrial oxidative metabolism and some other sources may place a heavy burden of oxidative damage on mtDNA and mutations of the mitochondrial genome are an important cause of the human diseases such as cancer, aging, and several other degenerative diseases, a better understanding of the mechanisms and pathways by which mtDNA is repaired may have significant implications in prevention or treatment of these disorders. Our findings reported here not only reveal a new function of Sirt3 but also provide a potential new paradigm for repair of mtDNA.

In recent years, accumulating evidence has pinpointed the existence of the repair machinery of the mitochondrial genome. For instance, the role of OGG1 in the repair of mtDNA has been well documented. ${ }^{30,31}$ Nevertheless, how the activity of this DNA repair enzyme is regulated remains less clear. A previous study by Bhakat et al. found that the function of OGG1 in the repair of the nuclear DNA is controlled by the p300-mediated acetylation of this DNA glycosylase. ${ }^{21}$ Consistently, in the current study, we found that the 8-oxoG incision activity of the mitochondrial OGG1 is also regulated by the acetylation level of the enzyme. Moreover, our experiments demonstrate that the acetylation of OGG1 is affected by the activity of Sirt3 and that the deacetylation catalyzed by Sirt3 stabilizes the OGG1 protein, thereby sustaining its capacity to repair mtDNA. The effects of Sirt3 on the acetylation and stability of OGG1 appear to be specific, as repression of another mitochondrial protein deacetylase, Sirt5, did not affect the acetylation and expression of OGG1 protein (Supplementary Figures $1 \mathrm{~A}$ and B). Also, silencing of Sirt5 expression had no effect on the radiation-induced apoptosis (Supplementary Figure 1C).

A number of studies have demonstrated the pivotal role of Sirt3 in the maintenance of the mitochondrial function and integrity. It was reported that through regulating ROS production or mitochondrial permeability transition pore or 
a

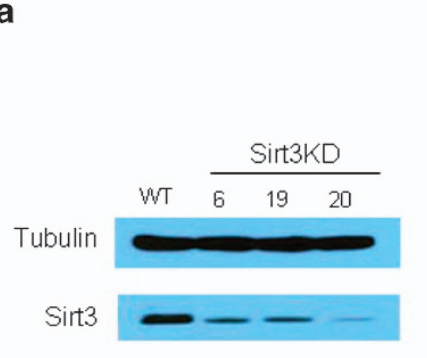

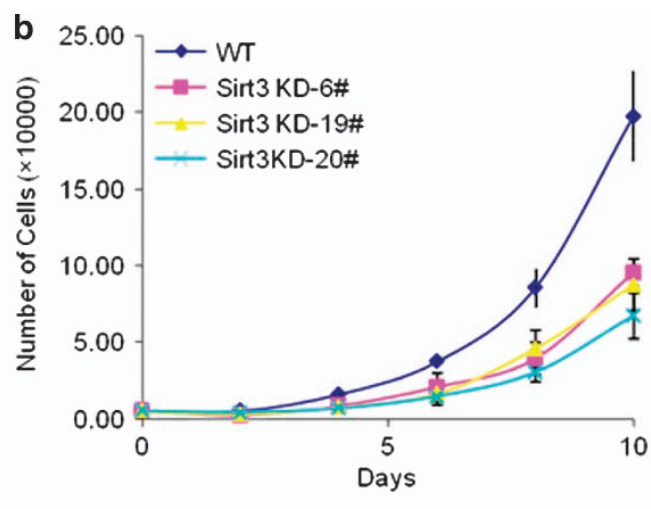

d
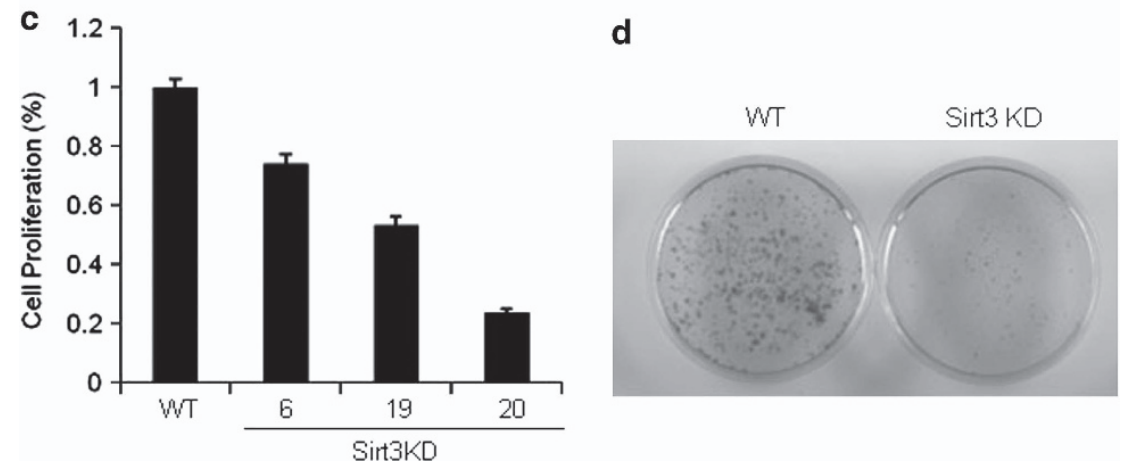

Figure 7 Expression level of Sirt3 is correlated with the ability of tumor cells to proliferate and form colony. (a) Western blot analysis of Sirt3 expression in LN229 cells expressing a Sirt3-targeted shRNA. Tubulin was used as a loading control. (b) Growth curves of LN229 cells expressing different levels of Sirt3. (c) LN229 cells expressing different levels of Sirt3 were seeded in a 96-well plate, and proliferation was determined using a BrdUrd incorporation assay kit. (d) LN229 wild-type and Sirt3-knockdown cells were plated in a six-well plate and incubated for 2 weeks at $37^{\circ} \mathrm{C}$. Cells were stained, and colonies were counted under a light microscope. "indicates clone number

by preventing Bax translocation to the mitochondria, Sirt3 protects various types of cells from apoptotic cell death triggered by genotoxic or oxidative stress. ${ }^{32-35}$ It is also known that the functions of the mitochondria are affected by the genomic integrity within this organelle. Oxidative damage to mtDNA can cause mitochondrial dysfunction and trigger apoptosis, which may be associated with the accumulation of 8-oxoG. ${ }^{36,37}$ We show in this study that by interacting with OGG1, Sirt3 plays an important role in protecting mtDNA from oxidative damage, as depletion of Sirt3 aggravates the irradiation-induced oxidative damage to mtDNA (Figure 4) and exacerbates the mitochondrial defects in the irradiated cells (Figure 5). These results are consistent with the report that overexpression of mitochondrial OGG1 blocks the angiotensin-induced mitochondrial dysfunction and apoptosis. ${ }^{38}$ Our study on the role of Sirt3 in mitigating mtDNA damage provides a new support to the notion that Sirt3 is a 'gatekeeper' of the mitochondrial integrity and function.

Owing to the discrepant results reported so far, the role of Sirt3 in cancer is still a question under debate. Although there are studies supporting pro-apoptotic and tumor suppressor roles of Sirt3, ${ }^{39,40}$ other reports claim that Sirt3 functions as a tumor promoter that contributes to survival of malignant cells. ${ }^{41}$ For example, it was reported that the Sirt3 ${ }^{-1-}$ mouse embryonic fibroblasts (MEFs) were more prone to develop tumors in nude mice than the Sir $3^{+1+}$ MEFs when Myc and Ras were introduced and that the Sirt $3^{-1}$ tumors grew faster and were larger than the Sir3 ${ }^{+1+}$ tumors. ${ }^{42}$ Also, Sirt3 expression was found to be lower in the tumor specimens from patients with breast cancer in comparison with the normal breast tissues. ${ }^{39}$ By contrast, there are studies showing that Sirt3 expression was increased in several types of cancer cell lines and tumor samples ${ }^{43,44}$ and that suppression of Sirt3 expression exerted an inhibitory effect on tumor cell growth and proliferation and sensitized tumor cells to chemotherapy and radiotherapy. ${ }^{41,43}$ Hence, whether Sirt3 is a tumor suppressor or a tumor promoter protein remains to be a controversial issue. Our finding that Sirt3 supports repairing of mtDNA in cells subjected to oxidative stress may help clarify this question. We propose that during tumor initiation, the protective role of Sirt3 in mtDNA may prevent genomic instability of the mitochondria in normal cells and this protects against carcinogenesis. On the other hand, expression of Sirt3 in cancer cells may impart resistance to 'environmental' or therapeutic stress, thereby contributing to the maintenance of the stability of cancer cells' mitochondrial genome and tumor survival and progression. It would be interesting to test whether therapeutic targeting of Sirt3 in cancer would suppress the malignant phenotypes such as invasion and metastasis and resistance to therapy.

Taken together, the results of our study demonstrate for the first time a role of Sirt3 in protecting mtDNA from oxidative damage and preventing apoptotic cell death and show that this protective role is mediated through the Sirt3-catalyzed deacetylation of OGG1. Thus, we not only identify the OGG1 as a new target for Sirt3 but also provide evidence supporting a new pathway of mtDNA repair. 
Materials and Methods

Cell lines and culture. The human glioblastoma cell lines T98G and LN229 and human embryonic kidney cell line HEK293T were purchased from American Type Culture Collection (Manassas, VA, USA). T98G cells were cultured in Ham's F-10/DMEM (10:1) medium, and LN229 and HEK293T cells were cultured in DMEM medium. The media were supplemented with $10 \%$ fetal bovine serum, $100 \mathrm{U} / \mathrm{ml}$ of penicillin, and $100 \mu \mathrm{g} / \mathrm{ml}$ of streptomycin. Cells were maintained at $37^{\circ} \mathrm{C}$ in a humidified atmosphere containing $5 \% \mathrm{CO}_{2} / 95 \%$ air.

Reagents and antibodies. Oligomycin (Olig), p-trifluoromethoxy carbonyl cyanide phenyl hydrazone (FCCP), 2-deoxyglucose (2DG), rotenone (Rote), ALLM, E64d, nicotinamide (NAM), CHX and Flag antibody were purchased from Sigma (Denver, CO, USA). Antibodies to Sirt3 and PARP were purchased from Cell Signaling Technologies (Boston, MA, USA). Acetyl-OGG1 antibody was purchased from Abcam (Cambridge, MA, USA). OGG1 and $\alpha$-tubulin antibodies were purchased from Santa Cruz Biotechnology (Dallas, TX, USA). All cell culture media were purchased from Invitrogen (Carlsbad, CA, USA). Western blot reagents were obtained from Pierce Biotechnology (Rockford, IL, USA).

siRNA, shRNA and plasmid transfection. siRNA targeting Sirt3 was purchased from Santa Cruz Biotechnology. Scrambled, non-targeting siRNA was used as a control. Transfection of siRNA was performed according to the manufacturer's protocol. Briefly, cells in exponential phase of growth were plated in six-well tissue culture plates at $1 \times 10^{5}$ cells per well, grown for $24 \mathrm{~h}$ and then transfected with siRNA using Oligofectamine and OPTI-MEM I-reduced serum medium. The concentrations of siRNAs were chosen based on dose-response studies.

shRNA targeting Sirt3 was purchased from Santa Cruz Biotechnology. LN229 cells were transfected with Sirt3 shRNA and then selected in $2 \mu \mathrm{g} / \mathrm{ml}$ of puromycin for 10 days. Multiple clones expressing Sirt3 shRNA were selected.

The Flag-Sirt3 plasmid was a gift from Dr. Yue Xiong (University of North Carolina at Chapel Hill, Chapel Hill, NC, USA), and the Flag-OGG1 plasmid was a gift from Dr. Bhakat and Dr. Mitra (University of Texas Medical Branch, Galveston, TX, USA) Transfection of the plasmid was carried out using FuGENE6 transfection reagent (Promega, Madison, WI, USA) according to the manufacturer's protocol.

In vitro deacetylation assay. HEK293T cells were transfected with a Flag-Sirt3 or a Flag-OGG1 plasmid, and the cells transfected with the Flag-OGG1 were treated with $10 \mathrm{mM}$ NAM. Then, the cells were lysed in lysis buffer $(50 \mathrm{mM}$ Tris-HCl (pH 7.4), $150 \mathrm{mM} \mathrm{NaCl}, 1 \mathrm{mM}$ EDTA, 1\% Triton X-100) supplemented with a protease inhibitor cocktail (Roche, Laval, QC, Canada) at room temperature for $20 \mathrm{~min}$, followed by centrifugation at $12000 \times \mathrm{g}$ for $20 \mathrm{~min}$. Flag-Sirt3 and Flag-OGG1 proteins were immunopurified separately using the ANTI-FLAG M2 affinity gel (Sigma) according to the manufacturer's protocol. For in vitro deacetylation assay, the purified OGG1-Flag protein was incubated with the Sirt3-Flag protein in the presence or absence of $1 \mathrm{mM} \mathrm{NAD}^{+}$in the deacetylase buffer ( $50 \mathrm{mM}$ Tris- $\mathrm{HCl}$ (pH 9.0), $4 \mathrm{mM} \mathrm{MgCl}, 50 \mathrm{mM} \mathrm{NaCl}, 0.5 \mathrm{mM}$ dithiothreitol) at $30^{\circ} \mathrm{C}$ for $3 \mathrm{~h}$, followed by western blot with an anti-OGG1 antibody. Expression of Sirt3 and OGG1 was shown as controls.

Western blot analysis. Cells were lysed in M-PER mammalian protein extraction reagent (Thermo Scientific, Rochester, NY, USA) supplemented with a protease inhibitor cocktail (Roche) at room temperature for $5 \mathrm{~min}$, followed by centrifugation at $14000 \times g$ for $10 \mathrm{~min}$. Lysates (20-40 $\mu \mathrm{g}$ protein) were resolved by SDS-PAGE and transferred to PVDF membrane. The PVDF membranes were then incubated with respective antibodies in $3 \% \mathrm{BSA} / \mathrm{TBST}$ at $4{ }^{\circ} \mathrm{C}$ for overnight, followed by incubation with a secondary antibody at room temperature for $1 \mathrm{~h}$. The protein signals were detected by the ECL method.

Immunoprecipitation. Cells were lysed in the IP lysis buffer $(150 \mathrm{mM}$ $\mathrm{NaCl}, 50 \mathrm{mM}$ Tris- $\mathrm{HCl}(\mathrm{pH} 7.4), 1 \%$ Triton $\mathrm{X}-100)$ in the presence of protease inhibitors (Roche) on ice for $5 \mathrm{~min}$, followed by centrifugation at $14000 \times g$ for $10 \mathrm{~min}$ at $4{ }^{\circ} \mathrm{C}$. Concentrations of proteins of the supernatants were determined, and samples containing equal amounts of proteins were precleared using protein $A / G$ plus-agarose beads (Santa Cruz Biotechnology) prior to immunoprecipitation. The antibodies were then added to the precleared lysates and incubated at $4{ }^{\circ} \mathrm{C}$ for $1-3 \mathrm{~h}$. After adding the fresh protein $\mathrm{A} / \mathrm{G}$ plus-agarose beads, the lysates were further incubated for overnight. Finally, the beads were washed three times with the lysis buffer, and the proteins were eluted using the sample loading buffer.
Preparation of substrate DNA and assay of DNA cleavage activity. The phosphoramidite for 8-0xoG was purchased from Glen Research (Sterling, VA, USA), and the oligodeoxynucleotide (5'-CCGGTGCATGACACTGT XACCTATCCTCAGCG- $3^{\prime}$ ) containing a single 8-OXOG ( $X$ in the sequence) was synthesized, purified by HPLC, and characterized by standard protocols. The oxoguanine glycosylase activity was measured using the standard strand-nicking assay. Briefly, $1 \mathrm{pmol}$ of ${ }^{32} \mathrm{P}$-labeled oxoG-containing 32 -mer oligonucleotide duplex was incubated with increasing amounts of mitochondrial lysate protein $(50,100,200$, and $500 \mu \mathrm{g})$ or purified hOGG1 in $10 \mathrm{mM}$ Tris- $\mathrm{HCl}(\mathrm{pH} 7.9), 50 \mathrm{mM}$ $\mathrm{NaCl}, 10 \mathrm{mM} \mathrm{MgCl}, 1 \mathrm{mM}$ DTT at $37^{\circ} \mathrm{C}$ for $30 \mathrm{~min}$. The reactions were terminated by incubation with proteinase $\mathrm{K}$ followed by phenol extraction. To promote complete strand cleavage at the abasic sites, the DNA samples were incubated with $0.2 \mathrm{~N} \mathrm{NaOH}$ at $95^{\circ} \mathrm{C}$ for $5 \mathrm{~min}$. The substrates and products were resolved by PAGE with $20 \%$ acrylamide containing $7 \mathrm{M}$ urea in $1 \times$ TBE buffer ( $89 \mathrm{mM}$ Tris, $89 \mathrm{mM}$ boric acid, $2 \mathrm{mM}$ EDTA) and visualized using a Typhoon 9200 .

Immunofluorescence imaging. Measurement of 8-0xoG amount in the mitochondria was performed as previously described. ${ }^{45}$ Briefly, the treated cells were cultured on glass coverslips and incubated with $200 \mathrm{nM}$ of MitoTracker Red (Invitrogen) at $37^{\circ} \mathrm{C}$ for $50 \mathrm{~min}$ in a humidified atmosphere containing $5 \% \mathrm{CO}_{2} /$ $95 \%$ air, followed by fixation with methanol at $-20^{\circ} \mathrm{C}$ for $10 \mathrm{~min}$. Then, the slides were incubated with $50 \mathrm{mM} \mathrm{NaOH}$ in $50 \%$ ethanol for $7 \mathrm{~min}$ at room temperature to denature the mtDNA. Following overnight incubation with an anti-8-oxoG antibody (Millipore, Billerica, MA, USA; 1:50 dilution), the cells were washed three times with TBST and then incubated with a fluorescein-conjugated secondary antibody (Cell Signaling Technologies; $1: 1000$ dilution) for $1 \mathrm{~h}$. The glass coverslips were finally mounted on microscope slides using an antifade medium containing DAPI (Life Technologies Co., Grand Island, NY, USA) and observed under a confocal microscope (Olympus, Tokyo, Japan; FluoView, FV1000).

DNA isolation and PCR. Total cellular DNA was isolated using DNeasy Blood and Tissue kit (Qiagen, Valencia, CA, USA) for PCR analysis. To detect the 4977-bp deletion in mtDNA, two pairs of nested primers were used:46 1F: AACCACAGTTTCATGCCCATC; 1R: TGTTAGTAAGGGTGGGGAAGC; 2F: ACCCTATTGCACCCCCTCTAC; and 2R: CTTGTCAGGGAGGTAGCGATG. The wild-type mtDNA primer sequences used were: forward GAAATGCCCCAAC TAAATACTACCGT; reverse AATGAGTGAGGCAGGAGTCCG. ${ }^{47}$ The PCR condition was set as: an initial denaturation at $95^{\circ} \mathrm{C}$ for $5 \mathrm{~min}$, followed by 30 cycles at $95^{\circ} \mathrm{C}$ for $10 \mathrm{~s}, 60^{\circ} \mathrm{C}$ for $30 \mathrm{~s}$, and $72{ }^{\circ} \mathrm{C}$ for $30 \mathrm{~s}$ and a final extension at $72{ }^{\circ} \mathrm{C}$ for $10 \mathrm{~min}$. PCR products were electrophoresed on a $2 \%$ agarose gel. Detection of wild-type mtDNA or the mtDNA 4977-bp deletion was evidenced by the appearance of a 438-bp and 358-bp band, respectively.

Measurement of oxygen consumption. The oxygen consumption was measured by: (1) real-time monitoring of oxygen consumption rates (OCRs) on a Seahorse XF24 Extracellular Flux Analyzer (Seahorse Bioscience Inc., North Billerica, MA, USA) according to the manufacturer's instruction. In these experiments, 10000 cells were seeded in the XF24 24-well plates in $150 \mu$ l of appropriate growth media and incubated overnight. Prior to measurements, cells were washed, immersed in $700 \mu \mathrm{l}$ of medium, and incubated in the absence of $\mathrm{CO}_{2}$ for $1 \mathrm{~h}$. OCRs were measured before and after addition of the metabolic inhibitors, Olig, FCCP, 2DG, and Rote. (2) Oxygen consumption was measured by an oxygen electrode with a water circulation system maintained at $37^{\circ} \mathrm{C}$. Briefly, $10 \times 10^{7}$ cells were collected by centrifugation at 800 r.p.m. and washed twice with $\mathrm{KCl}$ buffer $(140 \mathrm{mM} \mathrm{KCl}, 5 \mathrm{mM}$ $\mathrm{KH}_{2} \mathrm{SO}_{4}, 0.1 \mathrm{mM}$ EGTA). Cells were suspended in respiration buffer $(150 \mathrm{mM} \mathrm{KCl}$, $5 \mathrm{mM} \mathrm{KH}_{2} \mathrm{SO}_{4}, 5 \mathrm{mM} \mathrm{MgCl}$, $20 \mathrm{mM}$ HEPES, $0.05 \mathrm{mM} \mathrm{EGTA}$ ), and the cell suspension was added into the chamber. Let the pen sun and make the result.

Measurement of mitochondrial membrane potential. Mitochondrial membrane potential was measured by flow cytometry following JC-1 staining. Briefly, $4 \mu \mathrm{l}$ of Guava cell staining solution (including JC-1 and MitoPotential 7-AAD; Millipore) was added to $2 \times 10^{5}$ cells (in $200 \mu \mathrm{l}$ ), and the cells were incubated with the reagent for $30 \mathrm{~min}$ at $37^{\circ} \mathrm{C}$ in a humidified atmosphere containing $5 \% \mathrm{CO}_{2} / 95 \%$ air. At the end of incubation, the cells were analyzed by a Guava EasyCyte Plus FlowCytometry System.

ROS detection. ROS generation was measured using flow cytometry. Briefly, treated cells were collected and incubated with $10 \mu \mathrm{M}$ DCF-DA (Sigma) for $30 \mathrm{~min}$ 
at $37^{\circ} \mathrm{C}$ in a humidified atmosphere containing $5 \% \mathrm{CO}_{2} / 95 \%$ air. At the end of incubation, the samples were analyzed on an EasyCyte Plus FlowCytometry System.

Apoptosis assays. Apoptosis was determined by: (1) flow cytometric analysis of Annexin V and 7-AAD staining. Briefly, $100 \mu \mathrm{l}$ Guava Nexin reagent (Millipore) was added to $1 \times 10^{5}$ cells (in $100 \mu \mathrm{l}$ ), and the cells were incubated with the reagent for $20 \mathrm{~min}$ at room temperature in the dark. At the end of incubation, the cells were analyzed by a Guava EasyCyte Plus FlowCytometry System (Millipore). (2) Western blot analysis of PARP.

Cell proliferation assay. Cell proliferation was measured using the bromodeoxyuridine (BrdUrd) Cell Proliferation Assay Kit from Millipore, according to the manufacturer's instruction.

Clonogenic assay. LN229 wild-type or Sirt3-knockdown cells were plated in $35-\mathrm{mm}$ cell culture dishes (200 cells per dish), followed by incubation at $37^{\circ} \mathrm{C}$ in a humidified atmosphere containing $5 \% \mathrm{CO}_{2} / 95 \%$ air for 12 days. At the end of incubation, cells were stained with $1 \%$ methylene blue in $50 \%$ methanol for 30 min and washed with water, and colonies were counted.

Statistical analysis. The difference between the samples with or without silencing of Sirt3 was analyzed using an unpaired two-tailed Student's t-test. All experiments were performed at least three times.

\section{Conflict of Interest}

The authors declare no conflict of interest.

Acknowledgements. This study was supported by grants from the US Public Health Service R01CA135038 (J-M Yang), Elsa Pardee Foundation (J-M Yang), and the Department of Defense BC103654 (YC).

1. Finkel T, Deng CX, Mostoslavsky R. Recent progress in the biology and physiology of sirtuins. Nature 2009; 460: 587-591.

2. Lombard DB, Alt FW, Cheng HL, Bunkenborg J, Streeper RS, Mostoslavsky R et al. Mammalian Sir2 homolog SIRT3 regulates global mitochondrial lysine acetylation. Mol Cell Biol 2007; 27: 8807-8814.

3. Lu Z, Bourdi M, Li JH, Aponte AM, Chen Y, Lombard DB et al. SIRT3-dependent deacetylation exacerbates acetaminophen hepatotoxicity. EMBO Rep 2011; 12: 840-846.

4. Someya S, Yu W, Hallows WC, Xu J, Vann JM, Leeuwenburgh C et al. Sirt3 mediates reduction of oxidative damage and prevention of age-related hearing loss under caloric restriction. Cell 2010; 143: 802-812.

5. Schlicker C, Gertz M, Papatheodorou P, Kachholz B, Becker CF, Steegborn C. Substrates and regulation mechanisms for the human mitochondrial sirtuins Sirt3 and Sirt5. J Mol Biol 2008; 382: 790-801.

6. Cimen H, Han MJ, Yang $Y$, Tong $Q$, Koc H, Koc EC. Regulation of succinate dehydrogenase activity by SIRT3 in mammalian mitochondria. Biochemistry 2010; 49: 304-311.

7. Hallows WC, Lee S, Denu JM. Sirtuins deacetylate and activate mammalian acetyl-CoA synthetases. Proc Natl Acad Sci USA 2006; 103: 10230-10235.

8. Haigis MC, Deng CX, Finley LW, Kim HS, Gius D. SIRT3 is a mitochondrial tumor suppressor: a scientific tale that connects aberrant cellular ROS, the Warburg effect, and carcinogenesis. Cancer Res 2012; 72: 2468-2472.

9. Onyango P, Celic I, McCaffery JM, Boeke JD, Feinberg AP. SIRT3, a human SIR2 homologue, is an NAD-dependent deacetylase localized to mitochondria. Proc Natl Acad Sci USA 2002; 99: 13653-13658.

10. Qiu X, Brown K, Hirschey MD, Verdin E, Chen D. Calorie restriction reduces oxidative stress by SIRT3-mediated SOD2 activation. Cell Metab 2010; 12: 662-667.

11. Tao R, Coleman MC, Pennington JD, Ozden O, Park SH, Jiang H et al. Sirt3-mediated deacetylation of evolutionarily conserved lysine 122 regulates MnSOD activity in response to stress. Mol Cell 2010; 40: 893-904.

12. Chen $Y$, Zhang J, Lin $Y$, Lei $Q$, Guan KL, Zhao $S$ et al. Tumour suppressor SIRT3 deacetylates and activates manganese superoxide dismutase to scavenge ROS. EMBO Rep 2011; 12: 534-541.

13. Yu W, Dittenhafer-Reed KE, Denu JM. SIRT3 protein deacetylates isocitrate dehydrogenase 2 (IDH2) and regulates mitochondrial redox status. J Biol Chem 2012; 287: 14078-14086.

14. Ahn BH, Kim HS, Song S, Lee IH, Liu J, Vassilopoulos A et al. A role for the mitochondrial deacetylase Sirt3 in regulating energy homeostasis. Proc Natl Acad Sci USA 2008; 105: $14447-14452$.
15. Shulga N, Wilson-Smith R, Pastorino JG. Sirtuin-3 deacetylation of cyclophilin D induces dissociation of hexokinase II from the mitochondria. J Cell Sci 2010; 123(Pt 6): 894-902.

16. Schon EA. Mitochondrial genetics and disease. Trends Biochem Sci 2000 25: $555-560$

17. Richter C, Park JW, Ames BN. Normal oxidative damage to mitochondrial and nuclear DNA is extensive. Proc Natl Acad Sci USA 1988; 85: 6465-6467.

18. Grollman AP, Moriya M. Mutagenesis by 8-oxoguanine: an enemy within. Trends Genet 1993; 9: 246-249.

19. Cheng KC, Cahill DS, Kasai H, Nishimura S, Loeb LA. 8-Hydroxyguanine, an abundant form of oxidative DNA damage, causes G-T and A-C substitutions. J Biol Chem 1992; 267: $166-172$.

20. Roldan-Arjona T, Wei YF, Carter KC, Klungland A, Anselmino C, Wang RP et al. Molecular cloning and functional expression of a human CDNA encoding the antimutator enzyme 8-hydroxyguanine-DNA glycosylase. Proc Natl Acad Sci USA 1997; 94: 8016-8020.

21. Bhakat KK, Mokkapati SK, Boldogh I, Hazra TK, Mitra S. Acetylation of human 8-oxoguanine-DNA glycosylase by p300 and its role in 8-oxoguanine repair in vivo. Mol Cell Biol 2006; 26: 1654-1665.

22. Bohr VA, Stevnsner T, de Souza-Pinto NC. Mitochondrial DNA repair of oxidative damage in mammalian cells. Gene 2002; 286: 127-134.

23. Parlanti E, Fortini P, Macpherson P, Laval J, Dogliotti E. Base excision repair of adenine/8oxoguanine mispairs by an aphidicolin-sensitive DNA polymerase in human cell extracts. Oncogene 2002; 21: 5204-5212.

24. Takao M, Aburatani H, Kobayashi K, Yasui A. Mitochondrial targeting of human DNA glycosylases for repair of oxidative DNA damage. Nucleic Acids Res 1998; 26: 2917-2922.

25. de Souza-Pinto NC, Eide L, Hogue BA, Thybo T, Stevnsner T, Seeberg E et al. Repair of 8-oxodeoxyguanosine lesions in mitochondrial DNA depends on the oxoguanine DNA glycosylase (OGG1) gene and 8-oxoguanine accumulates in the mitochondrial DNA of OGG1-defective mice. Cancer Res 2001; 61: 5378-5381.

26. Minowa $\mathrm{O}$, Arai T, Hirano M, Monden $\mathrm{Y}$, Nakai S, Fukuda $\mathrm{M}$ et al. Mmh/Ogg1 gene inactivation results in accumulation of 8-hydroxyguanine in mice. Proc Natl Acad Sci USA 2000; 97: 4156-4161.

27. Hill JW, Hu JJ, Evans MK. OGG1 is degraded by calpain following oxidative stress and cisplatin exposure. DNA Repair (Amst) 2008; 7: 648-654.

28. Lee HC, Wei YH. Oxidative stress, mitochondrial DNA mutation, and apoptosis in aging. Exp Biol Med (Maywood) 2007; 232: 592-606.

29. Oka S, Ohno M, Tsuchimoto D, Sakumi K, Furuichi M, Nakabeppu Y. Two distinct pathways of cell death triggered by oxidative damage to nuclear and mitochondrial DNAs. EMBO J 2008; 27: 421-432.

30. Jensen A, Calvayrac G, Karahalil B, Bohr VA, Stevnsner T. Mammalian 8-oxoguanine DNA glycosylase 1 incises 8-oxoadenine opposite cytosine in nuclei and mitochondria, while a different glycosylase incises 8-oxoadenine opposite guanine in nuclei. J Biol Chem 2003; 278: 19541-19548.

31. Dobson AW, Xu Y, Kelley MR, LeDoux SP, Wilson GL. Enhanced mitochondrial DNA repair and cellular survival after oxidative stress by targeting the human 8-oxoguanine glycosylase repair enzyme to mitochondria. J Biol Chem 2000; 275 : 37518-37523.

32. Sundaresan NR, Samant SA, Pillai VB, Rajamohan SB, Gupta MP. SIRT3 is a stressresponsive deacetylase in cardiomyocytes that protects cells from stress-mediated cell death by deacetylation of Ku70. Mol Cell Biol 2008; 28: 6384-6401.

33. Chen CJ, Fu YC, Yu W, Wang W. SIRT3 protects cardiomyocytes from oxidative stressmediated cell death by activating NF-kappaB. Biochem Biophys Res Commun 2013; 430: 798-803.

34. Pellegrini L, Pucci B, Villanova L, Marino ML, Marfe G, Sansone $L$ et al. SIRT3 protects from hypoxia and staurosporine-mediated cell death by maintaining mitochondrial membrane potential and intracellular pH. Cell Death Differ 2012; 19: 1815-1825.

35. Shulga N, Pastorino JG. Ethanol sensitizes mitochondria to the permeability transition by inhibiting deacetylation of cyclophilin-D mediated by sirtuin-3. J Cell Sci 2010; 123(Pt 23): 4117-4127.

36. Rachek LI, Thornley NP, Grishko VI, LeDoux SP, Wilson GL. Protection of INS-1 cells from free fatty acid-induced apoptosis by targeting hOGG1 to mitochondria. Diabetes 2006; 55: $1022-1028$

37. Chatterjee A, Chang X, Nagpal JK, Chang S, Upadhyay S, Califano J et al. Targeting human 8-oxoguanine DNA glycosylase to mitochondria protects cells from 2-methoxyestradiol-induced-mitochondria-dependent apoptosis. Oncogene 2008; 27: $3710-3720$.

38. Ricci C, Pastukh V, Leonard J, Turrens J, Wilson G, Schaffer D et al. Mitochondrial DNA damage triggers mitochondrial-superoxide generation and apoptosis. Am J Physiol Cell Physiol 2008; 294: C413-C422.

39. Finley LW, Carracedo A, Lee J, Souza A, Egia A, Zhang J et al. SIRT3 opposes reprogramming of cancer cell metabolism through HIF1alpha destabilization. Cancer Cell 2011; 19: 416-428.

40. Bell EL, Emerling BM, Ricoult SJ, Guarente L. SirT3 suppresses hypoxia inducible factor 1alpha and tumor growth by inhibiting mitochondrial ROS production. Oncogene 2011; 30: 2986-2996.

41. Alhazzazi TY, Kamarajan P, Verdin E, Kapila YL. SIRT3 and cancer: tumor promoter or suppressor? Biochim Biophys Acta 2011; 1816: 80-88. 
42. Kim HS, Patel K, Muldoon-Jacobs K, Bisht KS, Aykin-Burns N, Pennington JD et al. SIRT3 is a mitochondria-localized tumor suppressor required for maintenance of mitochondrial integrity and metabolism during stress. Cancer Cell 2010; 17: 41-52.

43. Alhazzazi TY, Kamarajan P, Joo N, Huang JY, Verdin E, D'Silva NJ et al. Sirtuin-3 (SIRT3), a novel potential therapeutic target for oral cancer. Cancer 2011; 117 1670-1678.

44. Ashraf N, Zino S, Macintyre A, Kingsmore D, Payne AP, George WD et al. Altered sirtuin expression is associated with node-positive breast cancer. $\mathrm{Br} J$ Cancer 2006; 95: 1056-1061.

45. Ohno M, Oka S, Nakabeppu Y. Quantitative analysis of oxidized guanine, 8-oxoguanine, in mitochondrial DNA by immunofluorescence method. Methods Mol Biol 2009; 554: 199-212.
46. Chen T, He J, Shen L, Fang H, Nie H, Jin T et al. The mitochondrial DNA 4,977-bp deletion and its implication in copy number alteration in colorectal cancer. BMC Med Genet 2011; 12: 8.

47. Prithivirajsingh S, Story MD, Bergh SA, Geara FB, Ang KK, Ismail SM et al. Accumulation of the common mitochondrial DNA deletion induced by ionizing radiation. FEBS Lett 2004; 571: 227-232.

(c) (i) $\ominus$ Cell Death and Disease is an open-access journal published by Nature Publishing Group. This work is licensed under a Creative Commons Attribution-NonCommercialNoDerivs 3.0 Unported License. To view a copy of this license, visit http://creativecommons.org/licenses/by-nc-nd/3.0/

Supplementary Information accompanies this paper on the Cell Death and Disease website (http://www.nature.com/cddis) 